\title{
Giuseppe Ungaretti et le processus de création circulaire
}

La genèse de « Gridasti: soffoco »

\section{Emilio Sciarrino}

\section{OpenEdition}

\section{Journals}

Édition électronique

URL : http://journals.openedition.org/genesis/2429

DOI : 10.4000/genesis.2429

ISSN : 2268-1590

Éditeur :

Presses universitaires de Paris Sorbonne (PUPS), Société internationale de génétique artistique littéraire et scientifique (SIGALES)

\section{Édition imprimée}

Date de publication : 4 juin 2018

Pagination : 145-157

ISBN : 979-10-231-0604-6

ISSN : $1167-5101$

\section{Référence électronique}

Emilio Sciarrino, «Giuseppe Ungaretti et le processus de création circulaire », Genesis [En ligne], 46 | 2018, mis en ligne le 05 novembre 2018, consulté le 07 septembre 2019. URL : http:// journals.openedition.org/genesis/2429; DOI : 10.4000/genesis.2429 


\title{
Giuseppe Ungaretti et le processus de création circulaire La genèse de Gridasti : soffoco
}

\author{
Emilio Sciarrino
}

\section{Introduction}

Giuseppe Ungaretti représente un cas célèbre d'écrivain bilingue franco-italien ${ }^{1}$. Bien que ses textes publiés directement en français datent des deux premières décennies du $\mathrm{XX}^{\mathrm{e}}$ siècle $^{2}$, l'essentiel de son œuvre ${ }^{3}$ est en fait caractérisé par un incessant mouvement d'autotraduction entre les deux langues, qui s'accompagne de nombreux remaniements et variations ${ }^{4}$. Cependant, les éditions italiennes de référence ne mentionnent pas ce passage entre les langues 5 . Le processus de traduction et de variation a lieu aussi quand l'auteur, au lieu de s'autotraduire, collabore avec un traducteur. Ungaretti est également un traducteur renommé d'autres poètes ${ }^{6}$. Le dossier inédit que nous allons mettre au jour et analyser montre que, après avoir contribué à la traduction française d'un poème italien très personnel, Giuseppe Ungaretti revient sur le texte d'origine et le modifie à la lumière de cette expérience traductive, pour enfin le retraduire en français avec d'autres traducteurs. Dans cette genèse bilingue, les diverses étapes d'écriture, traduction, réécriture et retraduction suivent un parcours itératif qui va d'une langue à l'autre : nous proposons d'appeler ce phénomène le "processus de création circulaire».

Ce dossier, entièrement consacré aux échanges entre Ungaretti et le professeur italianiste Robert O. J. Van Nuffel, fait partie du fonds Van Nuffel qui est conservé aux Archives et Musée de la Littérature de Bruxelles ${ }^{7}$. Il recueille notamment plusieurs lettres, documents de travail et coupures de journaux qui permettent de retracer la genèse de la traduction du poème intitulé « Gridasti : soffoco» 8 [Tu crias : j'étouffe] qui a été réalisée en 1950 par Van Nuffel et qui a été revue par Ungaretti lui-même. Rappelons les circonstances de l'écriture du poème. En 1939, alors que Giuseppe Ungaretti et sa famille résident à Saõ Paulo, son fils Antonietto, âgé de neuf ans, meurt d'appendicite 9 . Le dernier cri de l'enfant à l'agonie aurait été «soffoco» [j'étouffe]. Cette expérience tragique marquera à jamais l'écrivain qui la mettra en lumière dans le poème «Gridasti : soffoco ». La douleur intime de la perte d'un être cher se joint à l'horreur collective de la guerre qui déchirera bientôt le monde pour une deuxième fois en ce $\mathrm{XX}^{\mathrm{e}}$ siècle. Ce double traumatisme personnel et historique est retranscrit dans Il Dolore, paru en 1947. Pourtant, le poème «Gridasti : soffoco», qui devait ouvrir la première section du recueil, Giorno dopo giorno, n'y est pas inclus lors de la publication. Ce n'est que deux ans plus tard que le poète décide de publier le poème «Gridasti : soffoco », d'abord en 1949 dans le journal Inventario, puis dans Il Popolo en 195010.

1. Un premier état de ces recherches a été présenté lors d'une communication au Congrès mondial de Traductologie, le 11 mars 2017 (en français).

2. Pour ses œuvres en français, voir notamment Giuseppe Ungaretti, Les Cinq Livres, texte établi en français par l'auteur et Jean Lescure, Paris, Les Éditions de Minuit, 1954 et Giuseppe Ungaretti, La Guerre, Jean-Charles Vegliante (éd.), Nantes, Le Passeur, 1999.

3. Giuseppe Ungaretti, Vita d'un uomo. Tutte le poesie, édité par Leone Piccioni, Milan, Mondadori, 1969.

4. Jean-Charles Vegliante, Ungaretti entre les langues, Paris, Presses de la Sorbonne Nouvelle, 1987.

5. Ibid., p. 15.

6. Luigi De Nardis, «Un parcours créatif : Ungaretti traducteur de Mallarmé », dans Genesis, n² 2, 1992, p. 49-60. Voir aussi : Isabelle Violante, Une cuvre originale de poésie, Giuseppe Ungaretti traducteur, Paris, Presses de l'université de Paris-Sorbonne, 1998.

7. Nous remercions les Archives et Musée de la Littérature de Bruxelles de nous avoir donné accès à ces documents et de nous avoir autorisé à les reproduire.

8. Giuseppe Ungaretti, « Gridasti: soffoco », dans Vita d'un uomo, op. cit., p. 263-264.

9. Cette maladie mal diagnostiquée pouvait à l'époque avoir une issue fatale. Sur cet épisode, voir Leone Piccioni, Vita di un poeta. Giuseppe Ungaretti, Milano, Rizzoli, 1970, p. 146-148. Voir aussi «Cronologia », dans Vita d'un uomo, op. cit., p. LX.

10. Voir les variantes dans l'apparat critique : Giuseppe Ungaretti, Vita d'un иoтo, op. cit., p. 813-816. Toutefois, cette édition ne mentionne aucunement la traduction. 
Très peu de temps après sa parution, Robert O. J. Van Nuffel, alors professeur d'italien à l'université de Gand, prend l'initiative de traduire «Gridasti : soffoco» [Tu crias : j'étouffe]. Il soumet alors par lettre le premier jet de sa traduction à Ungaretti, avec lequel il est déjà en contact. Le poète lui envoie des corrections en réponse, en mettant ainsi en place un dispositif de traduction collaborative que l'on retrouve chez de nombreux écrivains plurilingues ${ }^{11}$. Comme nous le verrons, cette traduction déçoit l'auteur, mais elle aura d'importantes conséquences pour la suite. En effet, après avoir remanié la traduction en français, le poète sera amené à retravailler, par ricochet, le texte en italien, exactement comme s'il s'autotraduisait ${ }^{12}$. Le texte italien remanié est finalement publié dans sa version définitive en 1952, dans le recueil Un grido e paesaggi [Un cri et des paysages] ${ }^{13}$ avant d'être retraduit en français par l'auteur en collaboration avec Jean Lescure en 1954, puis par Philippe Jaccottet en 197014 .

Ce dossier permet donc de reconstituer un processus créatif «circulaire» où l'on peut isoler trois étapes : en premier lieu, la traduction collaborative, puis un moment de réflexion solitaire (qui se rapproche de l'autotraduction), enfin, la réécriture du texte en italien qui conduira à son tour à de nouvelles traductions en français.

\section{La traduction collaborative Van Nuffel - Ungaretti}

Il est certain que Van Nuffel a lu «Gridasti : soffoco» dans Il Popolo, daté du 12 janvier 1950, car le dossier de Bruxelles comprend la coupure du journal que le traducteur a voulu conserver. C'est donc bien cette version qui lui a servi de point de départ. Nous la reproduisons ici :

1.

Non potevi dormire, non dormivi...

Gridasti : Soffoco...

Nel viso tuo scomparso già nel teschio,

Gli occhi, che erano ancora luminosi

Solo un attimo fa,

Gli occhi si dilatarono... Si persero...

Sempre ero stato timido,

Ribelle, torbido; ma puro, libero,

Felice rinascevo nel tuo sguardo...

Poi la bocca, la bocca
Che una volta pareva, lungo i giorni, Lampo di grazia e gioia,

La bocca si contorse in lotta muta... Un bimbo è morto...

2.

Nove anni, chiuso cerchio,

Nove anni cui né giorni, né minuti

Mai più s'aggiungeranno :

In essi s'alimenta

L'unico fuoco della mia speranza.

Posso cercarti, posso ritrovarti,

Posso andare, continuamente vado

A rivederti crescere

Da un punto all'altro

Dei tuoi nove anni.

Io di continuo posso,

Distintamente posso

Sentirti le mani nelle mie mani :

Le mani tue di pargolo

Che afferrano le mie senza conoscerle;

Le tue mani che si fanno sensibili,

Sempre più consapevoli

Abbandonandosi nelle mie mani;

Le tue mani che diventano secche

E - pallidissime! - nell'ombra, sole...

La settimana scorsa eri fiorente...

3.

Ti vado a casa a prendere il vestito, Poi nella cassa ti verranno a chiudere Per sempre. No, per sempre

11. Voir Anthony Cordingley and Céline Frigau Manning (dir.), Collaborative Translation: From the Renaissance to the Digital Age, London, Bloomsbury, 2016. Voir aussi Emilio Sciarrino, « La traduction avec 1'auteur », dans Le Plurilinguisme en littérature, Paris, Éditions des archives contemporaines, coll. «Multilinguisme, traduction, création», 2016, p. 148-162.

12. Dans ces cas, qui ont été amplement documentés pour divers écrivains bilingues, après avoir autotraduit son propre texte, l'auteur revient à la première version afin de la corriger sous l'influence de sa traduction. Voir par exemple «Voyage» de Ungaretti (dans La Guerre) et «Viaggio» dans Allegria di Naufragi ainsi que Jean-Charles Vegliante, Ungaretti entre les langues, op. cit., p. 15 et p. 4.

13. Giuseppe Ungaretti, Un grido e paesaggi, Milan, Schwarz, 1952.

14. Giuseppe Ungaretti, «Tu crias : j'étouffe», dans Les Cinq Livres, op. cit., p. 311-313 ; Giuseppe Ungaretti, «Tu as crié : j'étouffe», dans Vie d'un homme, traduit par Philippe Jaccottet, Paris, Les Éditions de Minuit/Gallimard, 1981, p. 266-268. (Première édition : 1970). 
Sei animo della mia anima, e la liberi.

Ora meglio la liberi

Che il tuo sorriso vivo non sapesse,

Se m'inciti a soffrire.

Provala ancora, accrescile la forza,

Se vuoi - sino a te, caro! - che m'innalzi

Dove si vive calmi, senza morte.

4.

Sconto, sopravvivendoti, l'orrore

Degli anni che t'usurpo,

E che ai tuoi anni aggiungo,

Demente di rimorso,

Come se, ancora tra di noi mortale,

Tu continuassi a crescere.

Ma cresce solo, vuota,

La mia vecchiaia odiosa.

Come ora, era di notte,

E mi davi la mano.

Dicevo spaventato :

- È troppo azzurro questo cielo australe,

È di stelle troppo gremito...

- Babbo, perché?

- Figliuolo mio, perché!

6.

(Cielo sordo, che scende senza un soffio,

Sordo che udrò continuamente opprimere

Mani tese a scansarlo...)

Après avoir lu ce texte, Van Nuffel envoie à Ungaretti le premier jet de sa traduction : Ungaretti lui répond immédiatement (fig. 1) 15 :

Caro amico,

Le sono grato di tutto.

Le mando con alcuni ritocchi la poesia.

In italiano sono poesie che hanno un suono gravissimo.

Un abbraccio dal suo

Ungaretti 16

Cette lettre contient une première mise en garde : «en italien, ce sont des poèmes qui ont une tonalité très grave». Ungaretti a joint à cette lettre un document de travail constitué par deux feuillets dactylographiés par Van Nuffel où il a ajouté ses propres corrections au stylo turquoise (fig. $2 \mathrm{a}$ et $2 b$ ).

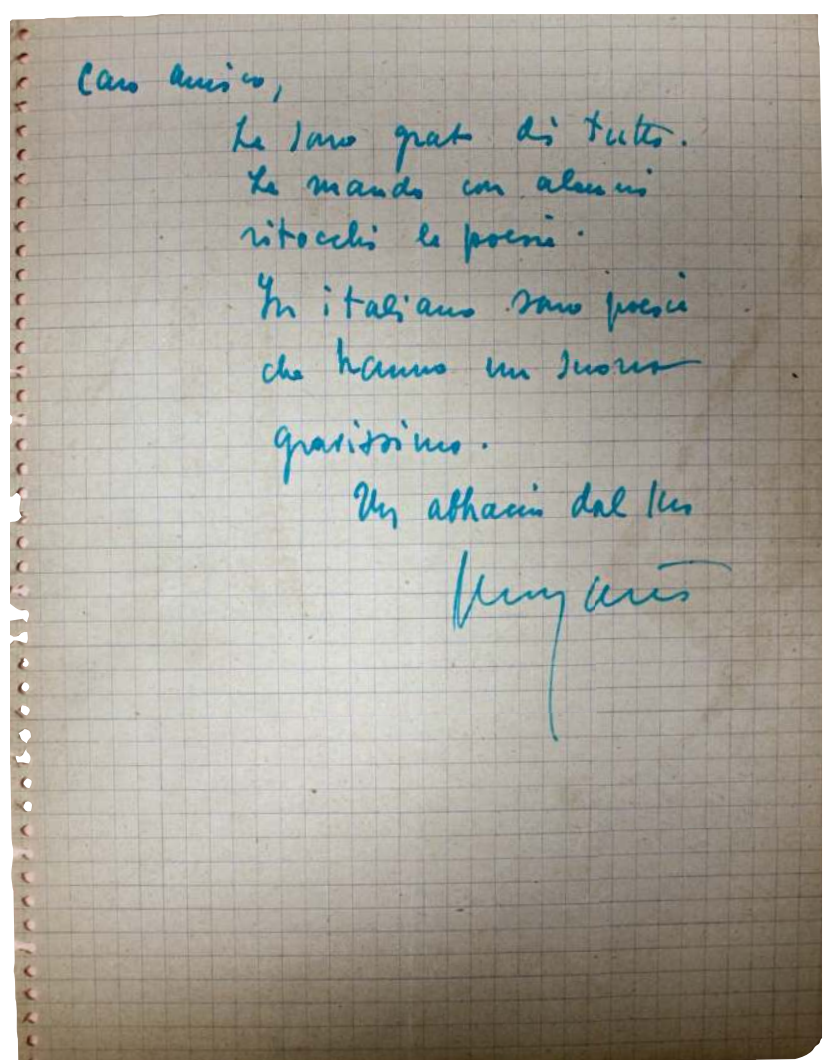

Fig. 1 : Lettre d'Ungaretti à Van Nuffel, non datée (1950)

Nous retranscrivons ici le texte en version semi-diplomatique. Les interventions d'Ungaretti sont signalées en gras.

\section{TU AS CRIÉ : JE SUFFOQUE.}

1.

Tu ne pouvais dormir, tu ne dormais pas...

Tu as crié : je suffoque..

Dans visage/ta figure/derrière/en en/le crâne déjà disparue,

Les yeux, qui étaient lumineux encor

15. Cette lettre n'est pas datée. L'enveloppe n'est pas conservée. 16. «Cher ami, je vous suis reconnaissant pour tout. Je vous envoie la poésie avec quelques retouches. En italien, ce sont des poésies qui ont une tonalité très grave. Je vous embrasse, Ungaretti. » Nous traduisons. 

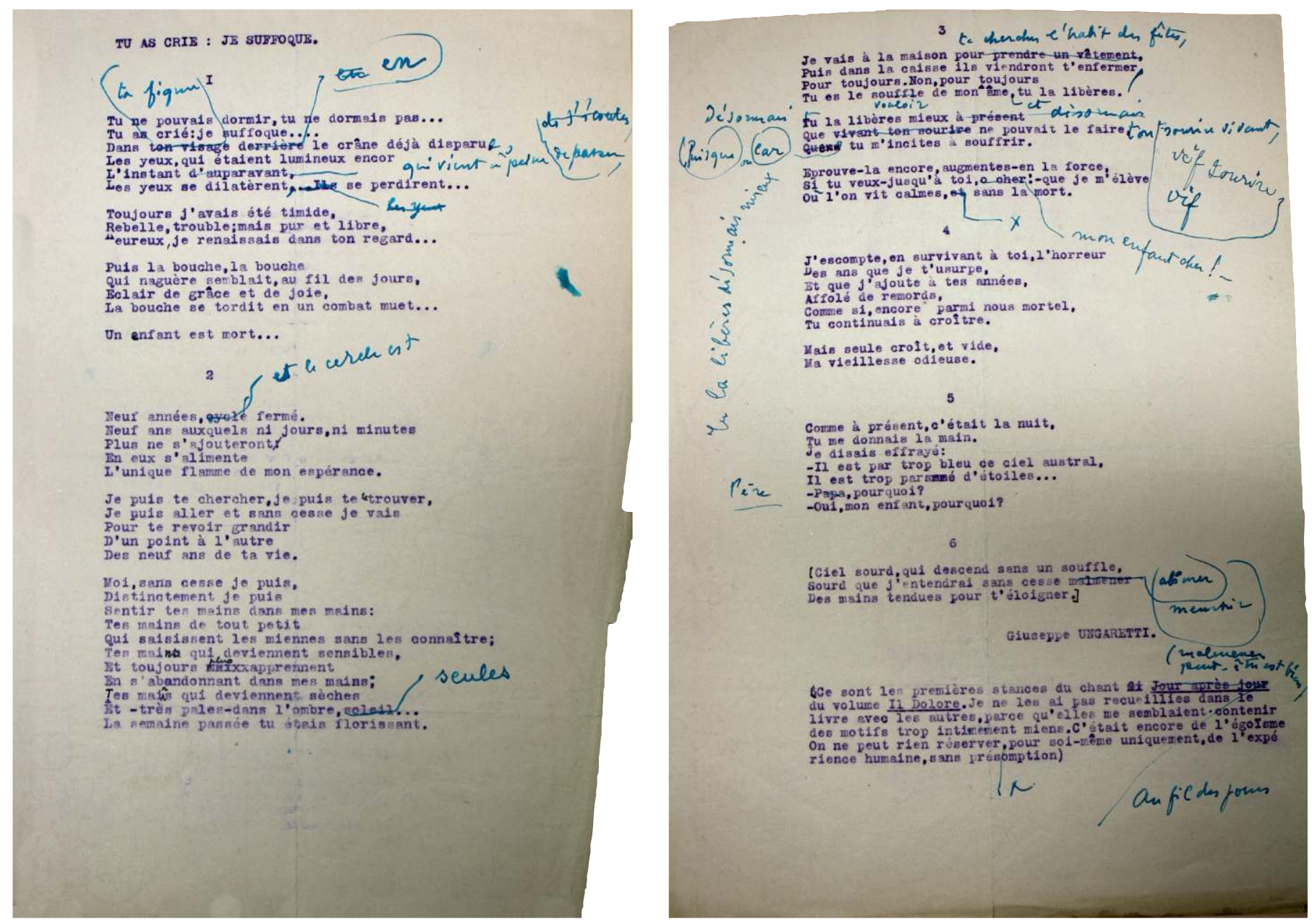

Fig. 2a et 2b : Traduction dactylographiée par Van Nuffel avec corrections manuscrites d'Ungaretti (2 feuillets)

L'instant d'auparavant,/qui vient à peine de passer, de s'écouler/

Les yeux se dilatèrent, Hs/les yeux/se perdirent...

Toujours j'avais été timide,

Rebelle, trouble; mais pur et libre,

Heureux, je renaissais dans ton regard...

Puis la bouche, la bouche

Qui naguère semblait, au fil des jours,

Éclair de grâce et de joie,

La bouche se tordit en un combat muet...

Un enfant est mort..

2.

Neuf années, eycle,/et le cercle est/fermé.

Neuf ans auxquels ni jours, ni minutes

Plus ne s'ajouteront :

En eux s'alimente

L'unique flamme de mon espérance.

Je puis te chercher, je puis te retrouver,

Je puis aller et sans cesse je vais

Pour te revoir grandir
D'un point à l'autre

Des neuf ans de ta vie.

Moi, sans cesse je puis,

Distinctement je puis

Sentir tes mains dans mes mains :

Tes mains de tout petit

Qui saisissent les miennes sans les connaître;

Tes mains qui deviennent sensibles,

Et toujours plus apprennent

En s'abandonnant dans mes mains;

Tes mains qui deviennent sèches

Et - très pâles - dans l'ombre, soleil.../seules/

La semaine passée tu étais florissant.

[2e feuillet]

3.

Je vais à la maison pour prendre un vêtement,/te chercher l'habit des fêtes,/

Puis dans la caisse ils viendront t'enfermer,

Pour toujours. Non, pour toujours

Tu es le souffle/vouloir/de mon âme/et/tu la libères. 
Désormais/Tu la libères mieux à présent désormais/Tu la libères désormais mieux/

Que vivant ton sourire ne pouvait le faire,/ton sourire si vivant,/vif sourire vif/

Quand/Puisque ou Car/tu m'incites à souffrir.

Éprouve-la encore, augmentes-en la force,

Si tu veux - jusqu'à toi,-o cher!/mon enfant cher!-/que je m'élève

Où l'on vit calmes, et sans la mort.

4.

J'escompte, en survivant à toi, l'horreur

Des ans que je t'usurpe,

Et que j'ajoute à tes années,

Affolé de remords,

Comme si, encore parmi nous mortel,

Tu continuais à croître.

Mais seule croît, et vide,

Ma vieillesse odieuse.

5 .

Comme à présent, c'était la nuit,

Tu me donnais la main.

Je disais effrayé :

- Il est par trop bleu ce ciel austral,

Il est trop parsemé d'étoiles..

- Papa/Père/, pourquoi?

- Oui, mon enfant, pourquoi?

6.

(Ciel sourd, qui descend sans un souffle,

Sourd que j'entendrai sans cesse malmener/abîmer/meurtrir/(malmener peut-être est bien)

Des mains tendues pour t'éloigner.)

\section{Giuseppe UNGARETTI.}

(Ce sont les premières stances du chant Jour après jourtAu fil des jours/du volume Il Dolore. Je ne les ai pas recueillies dans le livre avec les autres, parce qu'elles me semblaient contenir des motifs trop intimement miens. C'était encore de l'égoïsme. On ne peut rien réserver, pour soi-même uniquement, de l'expérience humaine, sans présomption).

Le lendemain, Ungaretti envoie une deuxième lettre où il indique quatre modifications, dont la première et la dernière sont déjà signalées sur les feuillets dactylographiés, alors que la deuxième et la troisième sont nouvelles (fig. 3) :

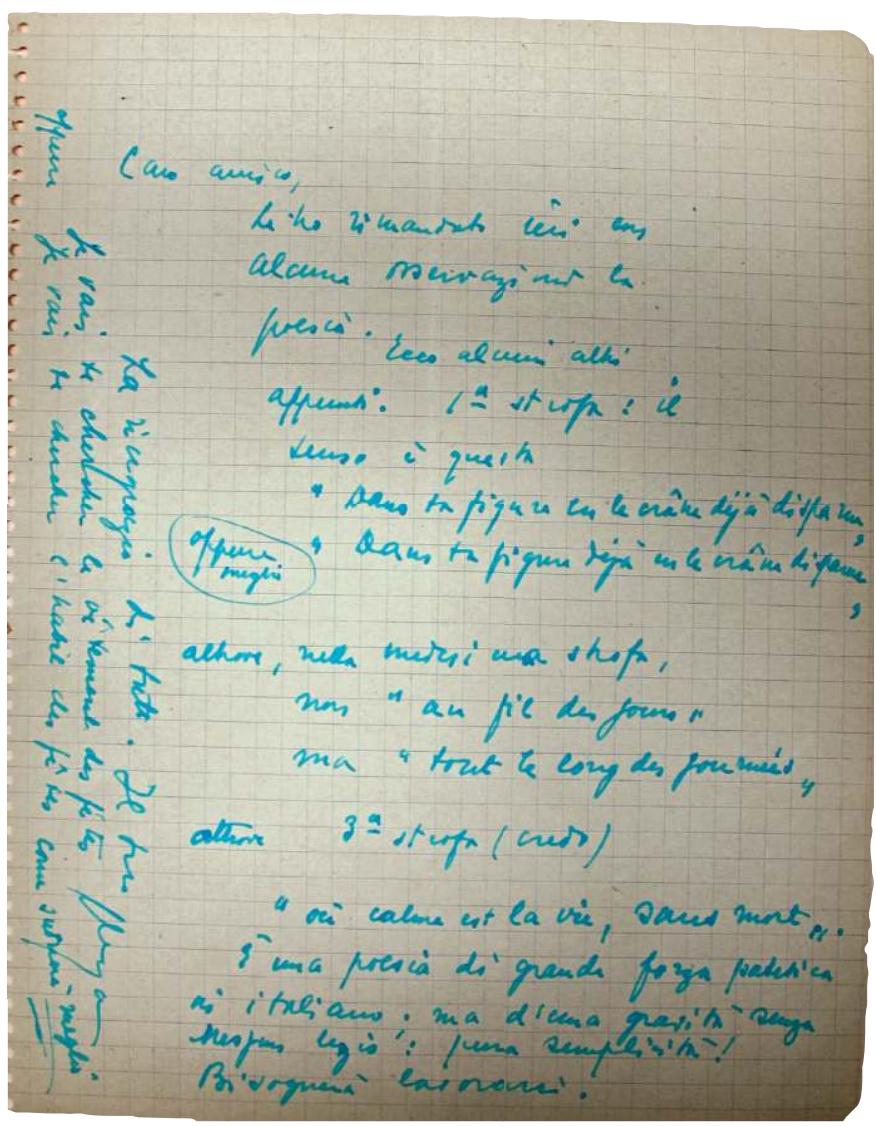

Fig. 3 : Lettre d'Ungaretti à Van Nuffel, non datée (1950)

Caro amico,

Le ho rimandato ieri con alcune osservazioni la poesia.

Ecco alcuni altri appunti.

1 a strofa : il senso è questo

«Dans ta figure en le crâne déjà disparue »

oppure meglio «Dans ta figure déjà en le crâne disparue»

altrove, nella medesima strofa,

non «au fil des jours»

ma «tout le long des journées »

altrove $3^{\text {a }}$ strofa (credo)

« où calme est la vie, sans mort».

È una poesia di grande forza patetica in italiano; ma d'una gravità senza nessun lezio : pura semplicità!

Bisognerà lavorarci.

La ringrazio di tutto

Il suo Ungaretti

Je vais te chercher le vêtement des fêtes

oppure Je vais te chercher l'habit des fêtes come suonerà meglio. 
Cher ami,

Je vous ai renvoyé hier mon poème avec quelques observations.

Voici quelques autres remarques.

1ère strophe : le sens est le suivant :

«Dans ta figure en le crâne déjà disparue»

ou mieux «Dans ta figure déjà en le crâne disparue »

ailleurs, dans la même strophe,

non pas «au fil des jours »

mais «tout le long des journées »

ailleurs 3 ème strophe (je crois)

«où calme est la vie, sans mort».

C'est un poème d'une grande force pathétique en italien, mais d'une gravité sans aucun artifice : pure simplicité !

Il faudra y travailler.

Je vous remercie de tout.

Votre Ungaretti

Je vais te chercher le vêtement des fêtes

ou Je vais te chercher l'habit des fêtes comme cela sonnera le mieux.

$*$

Là aussi, la lettre contient un avertissement : «c'est un poème d'une grande force pathétique en italien, mais d'une gravité sans aucun artifice : pure simplicité ! Il faudra y travailler.» Les corrections d'Ungaretti révèlent non seulement les carences de la traduction, mais aussi les points difficiles du texte. Par ailleurs, ses amendements sont systématiques et peuvent être répartis en quatre catégories : premièrement, des explicitations (ajouts qui n'étaient pas présents dans le texte d'origine), deuxièmement, des modifications des adverbes de temps, troisièmement des adaptations syntaxiques et, enfin, un important travail sur les nuances des substantifs. Entre autres, Ungaretti corrige un véritable contresens du traducteur : dans le vers «Le tue mani che diventano secche / E - pallidissime! - nell'ombra, sole» traduit par Van Nuffel «Tes mains qui deviennent sèches / Et - très pâles dans l'ombre, soleil...», «sole» n'est pas «soleil», mais bien «seules», adjectif qui qualifie les mains de l'enfant. Toutefois, que ce soit sur la version dactylographiée ou dans sa liste de corrections envoyée par lettre, il n'impose pas toujours des solutions univoques et laisse peut-être croire qu'il ménage un espace de liberté pour son traducteur. En réalité, il n'en est rien.
De son côté, après avoir reçu ces amendements, Van Nuffel corrige sa traduction et la publie très rapidement : elle paraît le 1er février 1950 dans Le Journal des poètes ${ }^{17}$. Voici le texte de la traduction :

\section{UN INÉDIT D’UNGARETTI}

TU AS CRIÉ : JE SUFFOQUE

1.

Tu ne pouvais dormir, tu ne dormais pas...

Tu as crié : je suffoque...

Dans ta figure dans le crâne déjà disparue,

Les yeux, qui étaient lumineux encor

L'instant à peine écoulé,

Les yeux se dilatèrent, ils se perdirent...

Toujours j'avais été timide,

Rebelle, trouble; mais pur et libre,

Heureux, je renaissais dans ton regard...

Puis la bouche, la bouche

Qui naguère semblait, au fil des jours...

Éclair de grâce et de joie,

La bouche se tordit en un combat muet...

Un enfant est mort...

Neuf années et le cercle est fermé.

Neuf ans auxquels ni jours, ni minutes

Plus ne s'ajouteront

En eux s'alimente

L'unique flamme de mon espérance.

Je puis te chercher, je puis te retrouver,

Je puis aller et sans cesse je vais

Pour te revoir grandir

D'un point à l'autre

Des neuf ans de ta vie.

Moi, sans cesse je puis,

Distinctement je puis

Sentir tes mains dans mes mains :

Tes mains de tout petit

Qui saisissent les miennes sans les connaître;

17. Le Journal des poètes, $\mathrm{n}^{\circ} 2$, $1^{\mathrm{er}}$ février 1950. 
Tes mains qui deviennent sensibles, Et toujours plus apprennent

En s'abandonnant dans mes mains,

Tes mains qui deviennent sèches

Et - très pâles - dans l'ombre seules...

La semaine passée tu étais florissant.

3.

Je vais à la maison te prendre l'habit de fête,

Puis dans la caisse ils viendront t'enfermer

Pour toujours. Non, pour toujours

Tu es le vouloir de mon âme et tu la libères.

Tu la libères désormais mieux

Que ne pouvait la faire ton vivant sourire,

Car tu m'incites à souffrir.

Éprouve-la encore, augmentes-en la force,

Si tu veux - jusqu'à toi, cher enfant! - que je m'élève

Où l'on vit calmes, sans la mort.

4.

J'escompte, en survivant à toi, l'horreur

Des ans que je t'usurpe,

Et que j'ajoute à tes années,

Affolé de remords,

Comme si, encore parmi nous mortel,

Tu continuais à croître.

Mais seule croît, et vide,

Ma vieillesse odieuse.

5.

Comme à présent, c'était la nuit,

Tu me donnais la main.

Je disais effrayé :

- Il est par trop bleu ce ciel austral,

Il est trop parsemé d'étoiles...

- Père, pourquoi?

- Oui, mon enfant, pourquoi?

6.

(Ciel sourd, qui descend sans un souffle,

Sourd que j'entendrai sans cesse malmener

Des mains tendues pour t'éloigner.)

(Traduit par R.O.J. Van Nuffel.)
(Ce sont les premières stances du chant Jour après jour $\mathrm{du}$ volume Il Dolore. Je ne les ai pas recueillies dans le livre avec les autres, parce qu'elles me semblaient contenir des motifs trop intimement miens. C'était encore de l'égoïsme. On ne peut rien réserver, pour soi-même uniquement, de l'expérience humaine, sans présomption).

Giuseppe UNGARETTI.

Alors que Van Nuffel a scrupuleusement respecté les corrections d'Ungaretti notées sur la version dactylographiée, il n'a pas inséré les deux corrections supplémentaires que le poète lui avait communiquées dans sa deuxième lettre.

\section{Réflexions d'Ungaretti sur sa traduction}

Resté sans nouvelles, Ungaretti écrit alors le 22 février 1950 une lettre de relance au traducteur : il n'est pas au courant de la parution de son poème et s'en inquiète (fig. 4).

Carissimo amico,

Le ho mandato per lettera raccomandata da Parigi la sua traduzione della poesia : «Gridasti: Soffoco»..., con, alcune osservazioni. È assolutamente indispensabile tenerne conto, è indispensabile ch'io riceva il testo riveduto della Sua traduzione. Si tratta della mia poesia alla quale tengo di più. Forse è la mia più bella. Nell' atrocità dei suoi termini ho posto tutto il mio cuore e la mia speranza. È poesia nuda, precisa : non è possibile giuocare al di là del termine che ho precisamente stabilito per ciascun vocabolo. Non deve essere pubblicata (la traduzione) senza ch'io l'abbia riveduta ancora una volta. Il testo italiano è perfetto.

Le sono grato di quanto ha fatto per me, per il suo lavoro affettuoso e straordinariamente efficace nell'interpretare una poesia tra le più difficili e vere.

Le invio molti saluti, con la speranza di rivederLa presto a Roma.

Il suo Giuseppe Ungaretti

Roma, 22.2.1950

Piazza Remuria, 3

Très cher ami,

Je vous ai envoyé par courrier recommandé depuis Paris votre traduction du poème «Gridasti: Soffoco »... avec 
Caritrimo amico,

the ho mandats in letena zaceomangath

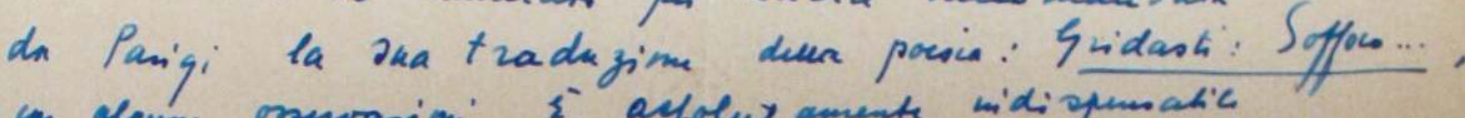

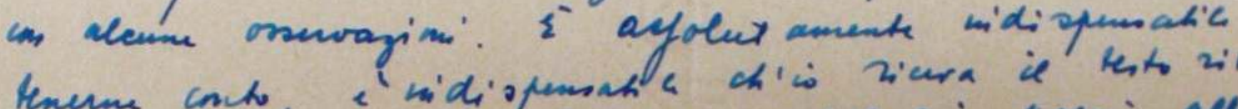

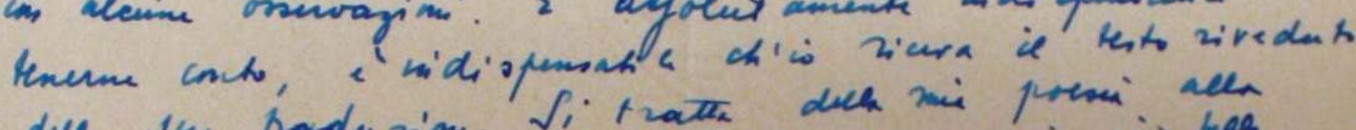

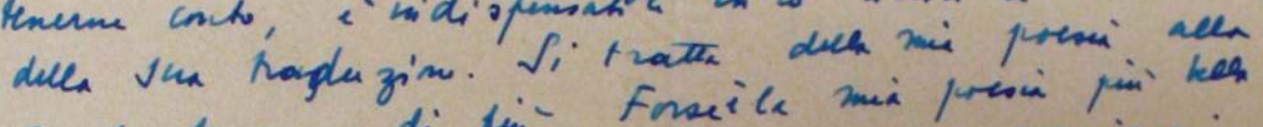

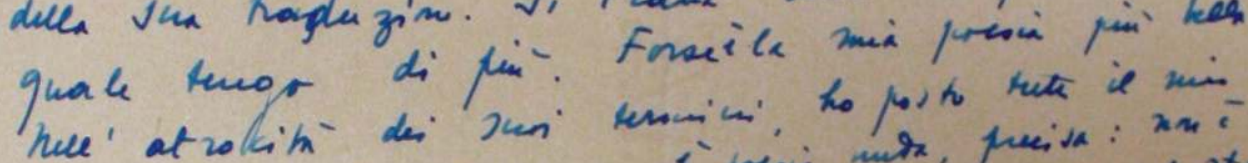

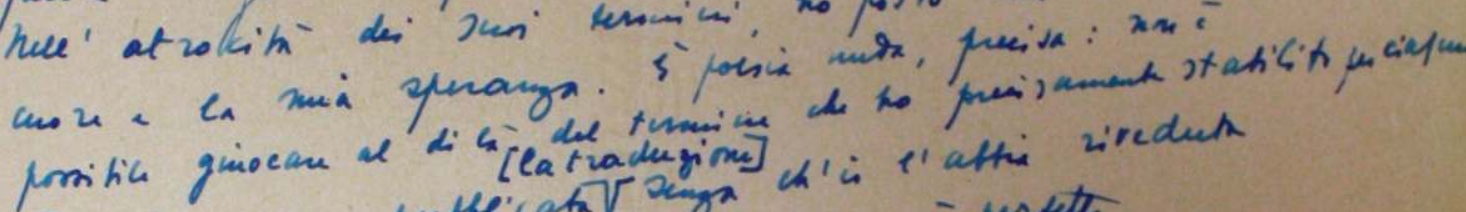

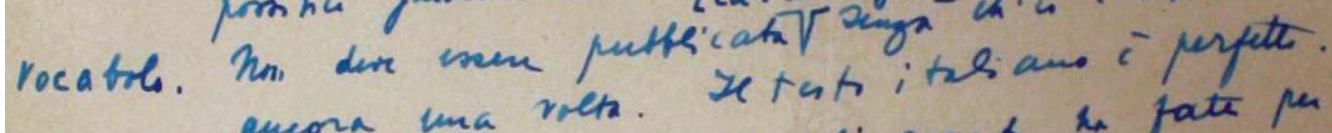

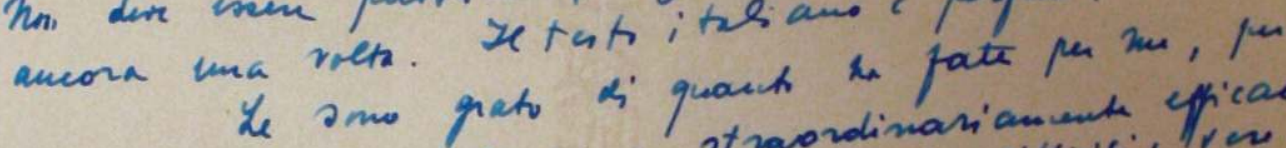

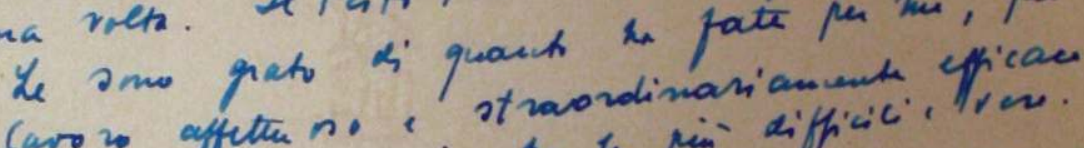

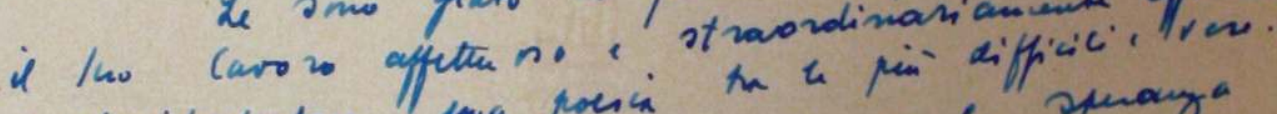

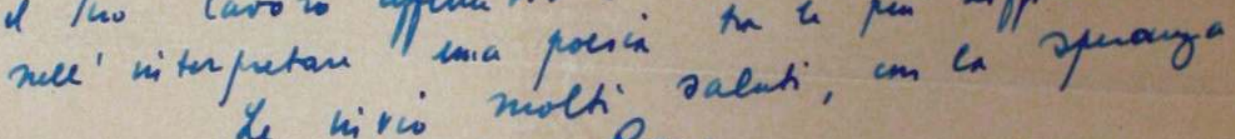

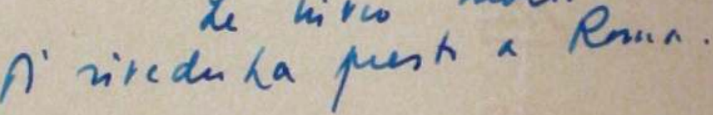
quisy" dengat

Rama, 22.2.1950. Piazza Remurin, 3

Fig. 4 : Lettre d'Ungaretti à Van Nuffel, le 22 février 1950 
quelques observations. Il est absolument indispensable d'en tenir compte, il est indispensable que je reçoive le texte revu dans sa traduction. C'est le poème auquel je tiens le plus. C'est peut-être mon plus beau texte. Dans l'atrocité de ses termes, j'ai mis tout mon cœur et tout mon espoir. C'est un poème nu, précis, il n'est pas possible de jouer au-delà de la limite que j'ai précisément établie pour chaque mot. La traduction ne doit pas être publiée sans que je l'aie revue encore une fois. Le texte italien est parfait.

Je vous suis reconnaissant de tout ce que vous avez fait pour moi, de votre travail affectueux et extraordinairement efficace dans l'interprétation d'un de mes poèmes les plus difficiles et les plus vrais.

Je vous envoie mes salutations, dans l'espoir de vous revoir bientôt à Rome.

Votre Giuseppe Ungaretti

Cette lettre dévoile avec plus de franchise la position de l'auteur : Ungaretti indique les limites de la traduction et de l'interprétation. En l'absence de documents, on pourrait multiplier les hypothèses sur les raisons du silence de Van Nuffel. On mentionnera que, exactement à la même époque, il était en train de traduire un autre grand poète italien, Eugenio Montale18... Deux semaines plus tard, Ungaretti revient à la charge, visiblement préoccupé, avec une autre lettre, datée du 7 mars 1950, où il récapitule une nouvelle fois ses corrections, toujours sans savoir que la traduction était parue un mois auparavant (fig. 5) :

Roma, Piazza Remuria, 3

il $7 / 3 / 1950$.

Caro amico,

Le ho scritto tre o quattro volte, e non ho ricevuto ancora nessuna risposta da parte Sua. Sono in pensiero. Subito da Parigi le ho fatto parvenire una mia lettera indicandole alcune sviste nella traduzione di «Gridasti : Soffoco...»

1) Dans ta figure, déjà disparue en le crâne

Tes yeux qui étaient, il n'y a qu'un instant, Encore lumineux

Les yeux se dilatèrent, s'égarent

Puis la bouche, la bouche,

Qui semblait une fois, tout au long des journées,
Éclair de grâce et de joie

2) Les mains qui deviennent/se font/sèches Et - très pâles - sont dans l'ombre, seules.

3) Je vais à la maison te chercher l'habit/le vêtement/des fêtes Tu es le vouloir de mon âme, et tu la libères

Tu la libères désormais mieux

Que ne savait le faire ton sourire vif

Si tu veux - jusqu'à toi, mon enfant, - que je m'élève Où calme est la vie, sans mort.

5) - Père, pourquoi ?

6) Ciel sourd, qui descend sans un souffle, Sourd que j'entendrai toujours abîmer Les mains tendues pour l'éviter tendues.

Sarebbe per me un grande dispiacere se venisse stampata senza che le sviste siano state emendate. Nessuno capirebbe nulla alla poesia. Le sono tanto tanto grato di quanto ha fatto per me. La saluto con molto affetto e spero di poterle presto far festa a Roma.

Il suo Giuseppe Ungaretti.

\section{$*$}

«Cher ami,

Je vous ai écrit trois ou quatre fois, et je n'ai toujours pas reçu de réponse de votre part. Je me fais du souci. Je vous ai immédiatement envoyé depuis Paris une lettre qui vous indiquait quelques erreurs dans votre traduction de «Gridasti : Soffoco...» [...] Je serais très peiné si elle était imprimée sans que les erreurs ne soient corrigées. Personne ne comprendrait rien à ce poème. Je vous suis tellement reconnaissant de ce que vous avez fait pour moi. Je vous salue avec beaucoup d'affection et j'espère y pouvoir bientôt fêter votre présence à Rome.»

Cette fois le ton est plus sec : il faut purement et simplement éliminer les «erreurs » qui rendraient ce poème incompréhensible. Par ailleurs, on remarquera que cette lettre présente encore quelques nouvelles variantes. Deux mois ont

18. En effet, le fonds Van Nuffel conserve un dossier recueillant les échanges avec Eugenio Montale (notamment une lettre de Montale à Van Nuffel datée du 9 mars 1950). Le poète indique dans ses lettres quelques corrections ponctuelles à ses traductions, mais il n'y a pas d'exemple de traduction dactylographiée corrigée de la main de l'auteur. 
$\begin{array}{lllllll}\text { G } & \text { E } & \text { N } & \text { E } & \text { S } & \text { I } & \text { S }\end{array}$

Rnua, Piaza Remunis, 3 il $7 / 3 / 1950$.

Caro Amines,

the his Shith the oquaters volte, i has

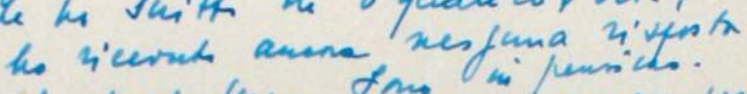
da parte Jus. Ino ho fate unvinite Inthits de Parigi he ho folle alecume tma hia etena, in $c_{i}$ in $d_{i}$ "gidasti: Joffoce...

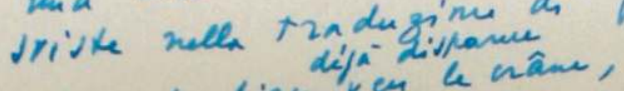

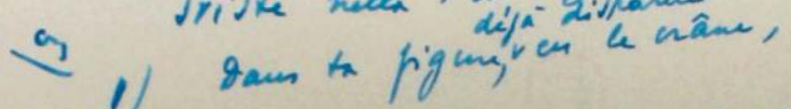

in your qui etaicht, is $x^{\prime} y^{a}$ qu'un instant,

Les your se dilationat, J'ígarent

Pini la fruch, is truch

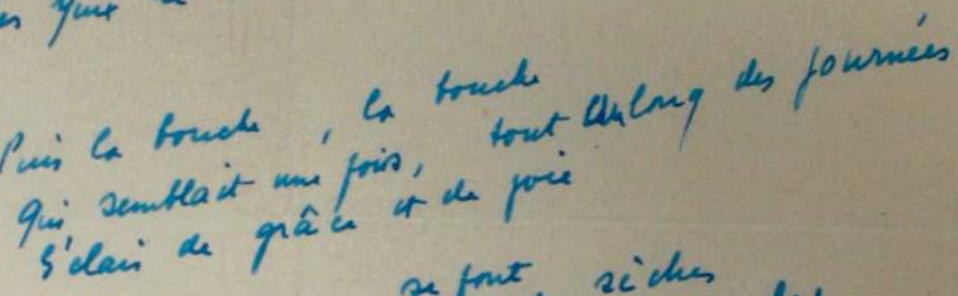

1) bn main qui dinimetent siches It - thes pales - 1 daus e'muse, seules.

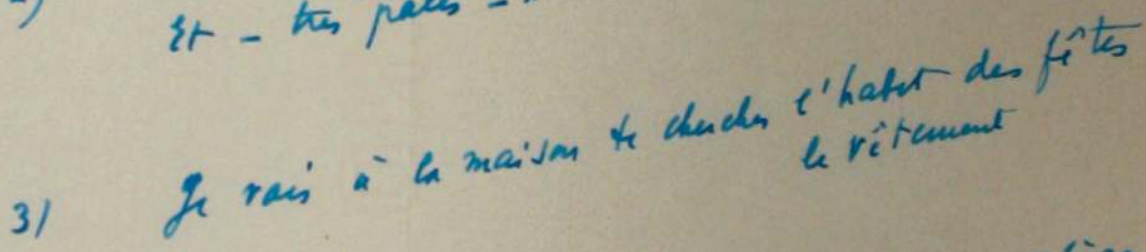

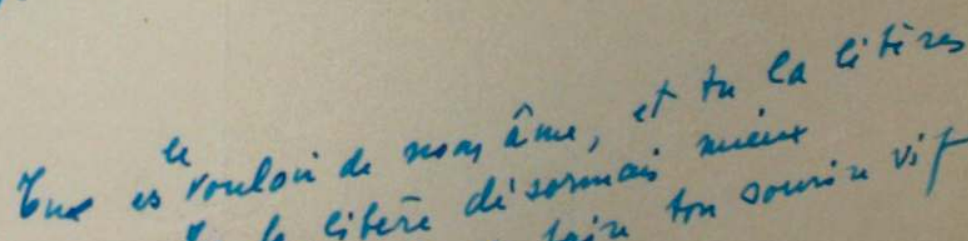

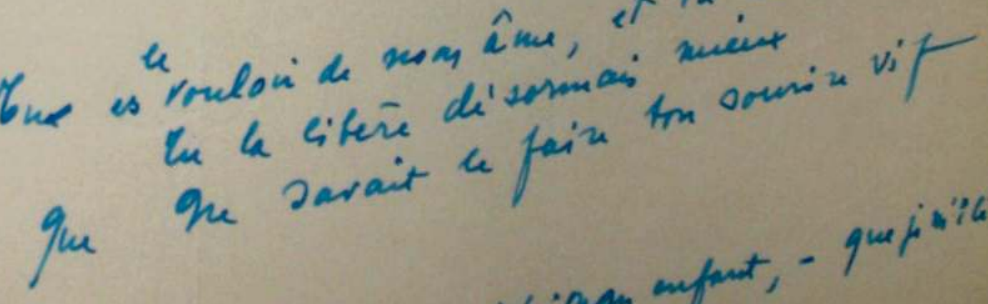

Si to rux - funqu'a'tripan mpent, - quefin'ilim

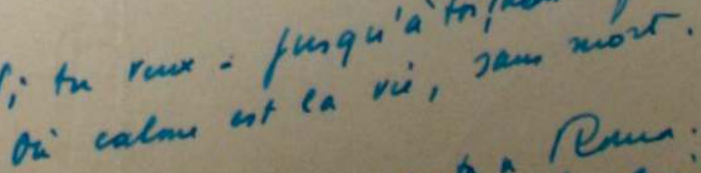

Fig. 5 : Lettre d'Ungaretti à Van Nuffel, le 7 mars 1950

(154)

Genesis 46.indb 154

$28 / 05 / 2018 \quad 13: 01$ 
passé depuis les premières corrections et le poète a pris du recul; il s'est émancipé de l'influence de son traducteur et il a affiné certaines de ses solutions, en effectuant un véritable travail de variation sur les termes français (par exemple, «il n'y a qu'un instant» au lieu de «l'instant d'auparavant», «s'égarèrent» au lieu de «se perdirent»). Ces dernières corrections resteront lettre morte et, à notre connaissance, il ne sera plus question de ce poème ${ }^{19}$.

\section{Réécriture du texte italien en vue de nouvelles traductions}

Cet épisode, à première vue anodin, a en fait des conséquences remarquables pour le devenir de «Gridasti: soffoco ». En effet, après avoir longtemps réfléchi à la traduction en français, Ungaretti remanie le texte en italien. Avant même de le publier en recueil, il le propose dès juillet 1951 à un autre traducteur et poète : Philippe Jaccottet. Comme le révèle la correspondance entre Ungaretti et Jaccottet, récemment publiée ${ }^{20}$, la lettre d'Ungaretti à son traducteur est accompagnée d'une version dactylographiée du poème (partiellement reproduite dans l'édition). Dans cette lettre, Ungaretti omet de préciser que son texte a déjà fait l'objet d'une traduction en français ${ }^{21}$. D' ailleurs, ce fait a échappé également à l'éditrice de la correspondance, qui commente simplement : «D'en remettre copie à Jaccottet pousse Ungaretti à trouver une meilleure distance avec ce texte et à se le réapproprier. Il en reprend la rédaction, lui apporte des modifications et réécrit toute la fin : le poème trouve alors sa forme définitive 22 ». Nous apporterons deux nuances : premièrement, à la lumière de ce dossier, nous savons qu'en réalité Ungaretti travaillait à ce poème depuis quelque temps, et qu'il l'avait déjà mis à l'épreuve à travers une première traduction. Deuxièmement, la version envoyée par Ungaretti en 1951 à Jaccottet a certes évolué par rapport à celle de 1950, mais elle ne coïncide pas avec la version finalement publiée en 1952. En tout cas, la traduction par Philippe Jaccottet mettra plus de temps que prévu à paraître.

Un an plus tard, en 1952, Giuseppe Ungaretti décide enfin de publier la version italienne de «Gridasti: soffoco » en recueil. Or, même s'il avait affirmé précédemment dans sa correspondance que «le texte italien est parfait», il apporte un certain nombre de modifications significatives à son texte italien dans son édition de 1952, qui présente la version définitive reprise à l'identique en 195423. Ces corrections sont visiblement influencées par l'expérience de la première traduction française en collaboration avec Van Nuffel, et sans doute apportées aussi en vue des nouvelles traductions en français à venir. En effet, on constate deux types de modifications. Certains remaniements concernent précisément les aspects qui avaient posé des difficultés lors de la traduction, comme si le poète voulait augmenter la «traduisibilité » de son texte. D'autres remaniements rapprochent le texte italien de sa première traduction française, notamment par rapport à l'ordre des mots ou aux changements syntaxiques.

Ainsi, on se souvient que les vers suivants avaient suscité un véritable contresens du traducteur :

Le tue mani che diventano secche

E - pallidissime! - nell'ombra, sole...

Tes mains qui deviennent sèches

Et - très pâles - dans l'ombre, soleil...

Van Nuffel avait traduit «sole»par «soleil» alors que cela signifie «seules», même si la position éloignée de

19. La dernière lettre présente dans le dossier témoigne d'un certain éloignement entre les deux hommes : Ungaretti s'y excuse de ne pas avoir pu trouver le temps de rencontrer Van Nuffel lors de la visite de ce dernier à Rome.

20. Voir la lettre de Giuseppe Ungaretti à Philippe Jaccottet, Rome, juillet 1951, dans Philippe Jaccottet-Giuseppe Ungaretti, Correspondance, édité par José-Flore Tappy, Paris, Gallimard, 2008, p. 45-46.

21. Ibid.

22. Ibid., note 1, p. 45.

23. L'apparat critique de Vita d'un uomo attribue les variantes à l'édition de 1954, mais en réalité celles-ci sont bien présentes dans le volume $U n$ grido e paesaggi paru chez Schwarz en 1952, dans le troisième volume de la collection de poésie «Campionario » dirigée par Giacinto Spagnoletti, avec une étude de Piero Bigongiari et cinq dessins de Giorgio Morandi, p. 25-27. En revanche, à la différence de l'édition de 1952, celle de 1954 est dédiée à Jean Paulhan. Le volume Un grido e paesaggi de 1952 a été imprimé à 340 exemplaires, plus dix exemplaires hors commerce. La bibliothèque Jacques Doucet à Paris en conserve un exemplaire, numéroté $n^{\circ} 333$, signé par Giuseppe Ungaretti et portant une dédicace manuscrite rédigée au stylo vert à Francis Ponge : «Très cher Ponge, je vais très prochainement dire dans Paragone toute l'admiration que m'inspire votre poésie. Bonne année, votre ami Giuseppe Ungaretti, Roma, il 23.12.1952». Ces multiples envois et dédicaces montrent encore une fois l'importance du public français aux yeux d'Ungaretti. 
l'article pouvait laisser planer le doute. Or, dans la nouvelle version italienne, l'adjectif «sole» est antéposé et répété deux fois, afin d'éviter toute ambiguiité :

Le tue mani che diventano secche

E, sole - pallidissime -

Sole nell'ombra sostano...

Tes mains qui deviennent sèches

Et, seules - très pâles -

Seules dans l'ombre se figent... ${ }^{24}$

Une autre modification concerne le vers « Che il tuo sorriso vivo non sapesse / Se tu m'inciti a soffrire», qui avait posé des problèmes, car il était assez elliptique : face à la traduction «Que vivant ton sourire ne pouvait le faire/quand tu m'incites à souffrir», Ungaretti avait d'abord changé l'ordre des mots du premier vers et pour traduire «si $s$ il avait remplacé «quand» par «car» ou «puisque». Visiblement, le lien logique entre les deux propositions restait difficile à expliciter. De plus, dans la lettre d'Ungaretti du 7 mars, le dernier état de la traduction est : «Que ne savait le faire ton sourire vif ». Or, dans la nouvelle version du poème en italien, le vers «se tu m'inciti a soffrire» [si tu m'incites à souffrir ] a été supprimé, et l'ordre des mots du vers restant a été changé pour reproduire celui de la version française : «Che non sapesse il tuo sorriso vivo» [Que ne savait le faire ton sourire vif].

Un autre exemple de transformation grammaticale calquée sur le français est le suivant : «Dove si vive calmi, senza morte», avait été traduit très littéralement par Van Nuffel : «où l'on vit calmes, sans mort», mais Ungaretti a proposé de transformer le verbe «on vit» en substantif : «où calme est la vie, sans la mort». Dans la version définitive, Ungaretti écrit en italien «Dove il vivere è calma, è senza morte», avec un infinitif substantivé en italien.

Enfin, la fin du poème a connu une modification substantielle. On se souvient que le père et son fils dialoguaient :

Dicevo spaventato :

- È troppo azzurro questo cielo australe,

È di stelle troppo gremito...

- Babbo, perché?

- Figliuolo mio, perché!

Je disais effrayé :

- Ce ciel austral est trop azur
Il est trop peuplé d'étoiles...

- Papa, pourquoi?

- Oui, mon petit enfant, pourquoi?

Or, tout ce passage a été radicalement modifié. Ce changement est sans doute lié à la difficulté de traduire deux termes, «babbo» et «figliuolo» 25 , qui avaient suscité des corrections de la part d'Ungaretti. La version envoyée à Philippe Jaccottet en 1951 contient déjà une version simplifiée du dialogue où ces deux termes disparaissent :

Dicevo spaventato :

- È questo cielo australe

Troppo gremito d'astri...

- Perchè?....

- Perchè?....26

Je disais effrayé :

- Ce ciel austral est

Trop peuplé d'astres...

- Pourquoi?...

- Pourquoi ?...

Dans la version définitive italienne, ce dialogue a tout simplement disparu, pour faire place à un monologue intérieur :

Spaventato tra me e me m'ascoltavo :

È troppo azzurro questo cielo australe,

Troppi astri lo gremiscono,

Troppi e, per noi, non uno familiare.

Effrayé je m'écoutais en mon for intérieur :

Ce ciel austral est trop azur,

Trop d'astres le peuplent,

Trop, et pour nous, pas un familier.

Enfin, si le texte italien de «Gridasti: soffoco» trouve sa fixation en 1952 lors de sa publication dans Un grido e paesaggi [Un cri et des paysages], le projet d'une traduction française tarde à aboutir. Même si Ungaretti avait déjà envoyé son texte à Philippe Jaccottet en juillet 1951, il se résout alors à traduire le poème avec Jean Lescure. Cette

24. Pour cet extrait et pour les suivants, nous donnons notre traduction. 25. Il s'agit de deux termes répandus essentiellement en Toscane. 26. Philippe Jaccottet-Giuseppe Ungaretti, Correspondance, op. cit., p. 45-46. 
traduction paraît en 1954 dans le recueil Les Cinq Livres 27 incluant à la fois des poèmes traduits de l'italien et des poèmes rédigés directement en français. La mention «texte établi en français par l'auteur et Jean Lescure» entretient justement une certaine ambiguïté sur la nature linguistique de ces textes... Quant à la traduction de Philippe Jaccottet, au demeurant fort différente, elle sera publiée seulement en 1970 , date de mort de l'auteur28 :

\section{TU AS CRIÉ : J'ÉTOUFFE}

Tu ne pouvais dormir, tu ne dormais pas...

Tu as crié : j'étouffe...

Dans ton visage avalé déjà par le crâne,

Les yeux, encore lumineux

Il n'y a qu'un instant,

Les yeux se dilatèrent... se perdirent...

Moi le timide, le trouble, le rebelle,

Dans ton regard je renaissais jadis

Pur, libre, heureux...

Puis la bouche, la bouche

Qui paraissait jadis, au long des jours,

Éclair de grâce et de joie,

La bouche se tordit, muette...

Un petit enfant est mort.

Neuf ans, cercle bouclé,

Neuf ans auxquels pas un jour

Ne s'adjoindra jamais plus :

Voilà le bois dont brûle

Mon seul espoir.

Je peux chercher, je peux te retrouver,

Aller je peux, je ne cesse d'aller

Te revoir qui grandis

Tout au long de tes neuf ans.

Moi sans cesse je peux,

Je peux distinctement

Sentir dans les miennes tes mains :

Tes menottes d'enfantelet

S'agrippant sans savoir aux miennes,

Tes mains qui se font sensibles,

Plus conscientes à mesure,

En s'abandonnant dans les miennes;

Tes mains qui deviennent sèches

Et seules - pâles à l'extrême,

Seules arrêtées dans l'ombre...

L'autre jour, tu resplendissais...
Je vais chercher tes beaux habits à la maison,

Ils vont venir t'enfermer pour toujours

Dans le cercueil. Non, pour toujours,

Tu es le souffle de mon âme, et la délivres !

La délivres mieux à présent

Que ne l'eût pu ton sourire vivant :

Éprouve-la encore, accrois sa force,

Si tu veux - jusqu'à toi - que je me hisse

Où vivre est calme, sans mort.

J'expie, en te survivant, l'horreur

Des années que je te vole,

Et je les ajoute aux tiennes,

Affolé par le remords,

Comme si, mortel encore parmi nous,

Tu continuais à grandir.

Mais seule grandit, stérile,

Ma vieillesse détestée...

C'était une nuit pareille,

Tu me donnais ta fine main...

Et moi, épouvanté, je m'entendais disant :

Ce ciel austral est trop bleu

Où pullulent trop d'astres

Et pas un de familier...

(Ciel sourd descendant sans un souffle,

Sourd que toujours j'entendrai écraser

Des mains tendues pour l'écarter...)

\section{Conclusion}

Du point de vue de l'histoire littéraire, la traduction française de Van Nuffel (1950) représente donc le «chaînon manquant» entre les premières versions du poème et la version publiée en 1952 qui donnera lieu à de nouvelles traductions en français. Même si cette première traduction en français, disparue de la mémoire collective, n'a pas satisfait Ungaretti, elle a laissé des traces dans le texte italien définitif. C'est en effet à partir de cette première ébauche

27. Giuseppe Ungaretti, «Tu crias : j'étouffe », dans Les Cinq Livres, op. cit., p. 311-313.

28. Giuseppe Ungaretti, «Tu as crié : j'étouffe », dans Vie d'un homme, op. cit., p. 266-268. 
que le poète est revenu sur sa version d'origine avant de la proposer à un nouveau traducteur français. Ainsi, la genèse de ce poème est représentative du processus d'écriture bilingue de Giuseppe Ungaretti, qui mettait le texte poétique à l'épreuve par un jeu de reflets, de variantes et de modulations en deux langues.

Ce dossier éclaire également la complexité de la création plurilingue, en particulier dans le domaine de la poésie. Nous y avons constaté une continuité, voire un enchevêtrement entre différents processus d'écriture : la création débouche sur une traduction collaborative qui prend des allures d'autotraduction pour enfin motiver un retour sur le texte premier en vue d'une nouvelle traduction. Loin d'être cloisonnées, toutes ces phases d'écriture participent d'un seul processus créatif que nous avons qualifié de «circulaire» et que l'on pourra retrouver sans doute chez d'autres auteurs plurilingues. Un des défis à venir pour les études sur le plurilinguisme littéraire sera donc de ne pas considérer chaque phénomène d'écriture séparément, mais de comprendre le système global de la genèse plurilingue. 